\title{
Development and characterization of ceftriaxone-loaded P3HB-based microparticles for drug delivery
}

\author{
Anastasiya V. Murueva ${ }^{a}$, Anna M. Shershneva ${ }^{\mathrm{b}}$, Kseniya V. Abanina ${ }^{\mathrm{b}}$, Svetlana V. Prudnikova ${ }^{\mathrm{b}}$, \\ Ekaterina I. Shishatskaya ${ }^{\mathrm{a}, \mathrm{b}}$ \\ ${ }^{a}$ Institute of Biophysics SB RAS 50/50 Akademgorodok, Krasnoyarsk, 660036, Russia \\ ${ }^{b}$ Siberian Federal University 79 Svobodny, Krasnoyarsk, 660041, Russia \\ *Corresponding author E-mail address: goreva_a@mail.ru
}

\begin{abstract}
In this study polymer-based microparticles are used to improve the therapeutic properties of ceftriaxone (CEF) and render them safer. Poly-3-hydroxybutyrate (P3HB) and poly-3hydroxybutyrate/polyethylene glycol (P3HB-PEG)-based microparticles were prepared by two methods: a double emulsification technique and spray-drying. The microparticles were characterized in terms of size and zeta potential, morphology, total drug loading and drug release. The microparticles had spherical shapes with diameters of a size range from 0.74 to 1.55 $\mu \mathrm{m}$ (emulsification technique) and from 3.84 to $6.51 \mu \mathrm{m}$ (spray-drying); the encapsulation efficiency of CEF was around $63 \%$ and $49 \%$ for these methods respectively. The CEF release from microparticles obtained by spray-drying reached $100 \%$ after 150 hours, while for microparticles obtained by emulsification technique the total release of CEF did not exceed $34 \%$ after 312 hours. Antibacterial activity of the microparticles was evaluated against gram-positive and gram-negative bacterial strains. In general, the encapsulation of ceftriaxone in polymeric microparticles preserves the therapeutic efficacy of the CEF and provides a prolonged effect.
\end{abstract}

Keywords: ceftriaxone, $\mathrm{PH} 3 \mathrm{~B}-$ microparticles, emulsification technique, spray-drying, drug release, antibacterial activity

\section{Introduction}

Scientific research and development of innovative drug delivery systems is a rapidly developing area worldwide. This trend is going to be strengthened in the future, as the cost of health care requires reducing costs and improving the effectiveness of existing dosage forms. Currently, we observe the crisis of antibiotic therapy not only due to a large number of resistant microorganisms, but also due to the lack of drugs that do not have resistance of one or other pathogens. Moreover, bacteria appeared that are resistant to all existing antibiotics. In the past the pharmaceutical industry solved the problem of resistance by producing a new, more effective antibiotic. However, today there are no fundamentally new classes of antibiotics that are acceptable for clinical use and the development of new drugs usually takes about 10-15 years (Zhang Y. et al., 2013). Therefore, the interest of the pharmaceutical sciences is gradually 
shifting from the discovery of new chemicals to the optimization of their routes of administration and delivery.

Thus, one of the drawbacks of classical antimicrobial therapy is that many intracellular bacteria are at rest or still, deactivating and dramatically changing the permeability of cell membranes, which affects the sensitivity to antibacterial agents. Therefore, such bacteria can persist for a long time. In addition, the most severe complications of antibiotic therapy are anaphylactic shock, allergic reactions, neurotoxic phenomena, dysbiosis, liver damage (Wright et al., 2013).

One of the promising ways to solve this problem is the use of antibacterial drugs in the form of micro- and nanocarriers. Micro- and nanoparticles based on biopolymers are very convenient drug carriers. By placing antibiotics in carriers we can expect improved delivery to infected cells, increased bioavailability of drugs with poor absorption characteristics, prolonged drug residence time, targeted transport of therapeutic agents to specific organs, reduced toxicity and stability in the gastrointestinal tract (Raval et al., 2012; Xie et al., 2014; Hamzehloo et al., 2017). A significant portion of antibiotics during the first 6 to 8 hours is mostly removed from the body. The use of microparticles allows to extend the elimination time (Xie et al., 2014; Zazo et al., 2016).

Ceftriaxone $(\mathrm{CEF})$ is a third-generation cephalosporin antibiotic that is effective mostly against gram-positive and gram-negative bacteria. $\mathrm{CEF}$ is a $\beta$-lactamase-resistant cephalosporin with a poor cellular penetration. Ceftriaxone is administered by slow intravenous infusion since it is poorly absorbed from gastrointestinal tract. (Manimekalai et al., 2017, Lipinski et al., 2001; Reddy et al., 2013; Shiffman et al., 1990). The literature describes examples of delivery systems for cephalosporin based on various materials. Thus, Kumar et al., 2016 demonstrated the efficacy of ceftriaxone-loaded solid lipid nanoparticles for gram-positive and gram-negative bacterial strains. In another paper the authors noted the pronounced antibacterial effect of conjugates of silver nanoparticles with ceftriaxone in the culture of various pathogenic strains of bacteria (Harshiny et al., 2015). Also, examples of prolonged forms of ceftriaxone in the form of conjugates based on poly (styrene-alt-maleic) anhydride (Najafi Moghadam et al., 2010), PEGylated microscopic lipospheres (Attama et al., 2009) and chitosan nanoparticles (Zaki et al., 2012).

Among the huge variety of polymers used to produce nano / microparticles, polyhydroxyalkanoates (PHA) has been the most widely used polymer in controlled release applications, including the encapsulation of different antimicrobial drugs (Li et al., 2005; Wang et al., 2007; Goreva et al., 2012; Vilos et al., 2012; Rodriguez-Contreras et al., 2013; Shershneva et al., 2014; Chen et al., 2015). This class of polyester attracts attention due to the presence of 
unique properties - biodegradation and biocompatibility. PHAs are thermoplastic, have less effect on $\mathrm{pH}$ values of tissues and have a longer in vivo degradation period, which allows them to be used for the development of prolonged drug delivery systems. It should be noted that spray drying is a popular way of depositing drugs in various polymer carriers, but with respect to PHA this method has not been properly developed. Single examples of the use of spray drying for producing microparticles based on polyhydroxybutyrate $(\mathrm{P} 3 \mathrm{HB})$ loaded with paracetamol $(\mathrm{Re}$ 2006). However, publications on the preparation of PHA-based antibiotic-loaded microparticles by spray drying method have rarely been seen until now.

Earlier we showed examples of the successful encapsulation of various antibacterial drugs in microparticles from $\mathrm{P} 3 \mathrm{HB}$ and copolymers with $\mathrm{P} 3 \mathrm{HB} / 3 \mathrm{HV}$ using the emulsion method (Goreva et al., 2012; Murueva et al., 2013). For the first time, the possibility of encapsulating an antitumor drug in a $\mathrm{P} 3 \mathrm{HB}$ microparticle was demonstrated using the spray drying method (Murueva et al., 2016, Shershneva et al., 2018). The dependences of the characteristics of the microparticles (yield, average diameter, zeta potential) on the parameters of the production process (inlet temperature, polymer solution feed rate and polymer solution concentration) are established (Shershneva et al., 2014).

Thus, the main objective of the study was to develop ceftriaxone delivery systems of as the polymeric microparticles. Microparticles from $\mathrm{P} 3 \mathrm{HB}$ and blend with polyethylene glycol (PEG) were produced by a spray-drying technique and emulsion method. Furthermore, to evaluate the effects of these methods and chemical composition on the properties of the microparticles CEF release in vitro. Besides, cytotoxicity and antibacterial efficiency of CEF encapsulated PHA microparticles were also investigated in vitro.

\section{Materials and Methods}

\section{Materials}

P3HB with low molecular weight was produced at the Institute of Biophysics of the SB RAS by the microbial fermentation process (Table 1). The registered mark of material is "Bioplastotan $^{\mathrm{TM}}$ ". PEG (35000 Da) was purchased Sigma-Aldrich (USA), ceftriaxone - FarmCenter (Russia) (Table 1).

Table 1. Materials and drug used in the work

\begin{tabular}{|c|c|c|c|}
\hline Material & Formula & $\begin{array}{l}\text { Molecular } \\
\text { weight, Da }\end{array}$ & Manufacturer \\
\hline P3HB & & 50000 & $\begin{array}{l}\text { Institute of Biophysics, } \\
\text { Siberian Branch, Russian } \\
\text { Academy of Sciences } \\
\text { (RF Patent No. 2051967) }\end{array}$ \\
\hline
\end{tabular}




\begin{tabular}{|l|l|l|l|}
\hline PEG & & 35000 & Sigma-Aldrich (USA) \\
\hline CEF & & Farm-Center (Russia) \\
\hline
\end{tabular}

\section{Preparation of microparticles by emulsion method (MP $\left.{ }_{E M}\right)$}

Microparticles were prepared by the solvent evaporation technique, using (oil/water) emulsions. The emulsion contained $0.1 \mathrm{~g}$ P3HB or P3HB/PEG (50:50) in $10 \mathrm{ml}$ of dichloromethane and $100 \mathrm{ml} 0.5 \%(\mathrm{w} / \mathrm{v})$ PVA. The obtained emulsions were mechanically stirred at 24,000 rpm during $5 \mathrm{~min}$ (IKA Ultra-Turrax T25 digital high-performance homogenizer, Germany). All emulsions were continuously mixed mechanically for 24 hours until the solvent completely evaporated. Microparticles were collected by centrifuging (at 10,000 rpm, for $5 \mathrm{~min}$ ), rinsed 4 times in distilled water and lyophilic dryer in an Alpha 1-2 LD plus (Christ, Germany).

The described above method was also used for loading CEF into microparticles. The CEF $(10 \mathrm{mg}$ ) was dissolved in $1 \mathrm{ml}$ of distillated water and added to the solution of $0.1 \mathrm{~g}$ P3HB or $\mathrm{P} 3 \mathrm{HB} / \mathrm{PEG}$ (50:50) in dichloromethane and the resulting emulsion was sonicated at $6 \mathrm{~W}$ for 2 min (Misonix 3000, USA). Then obtained emulsions were added to $0.5 \%$ (w/v) PVA solution at stirring at 24,000 rpm (IKA Ultra-Turrax T25 digital high-performance homogenizer, Germany). Centrifugation and washing conditions were similar to as described earlier.

\section{Preparation of microparticles by spray-drying $\left(M P_{S D}\right)$}

Spray-dried microparticles were prepared from P3HB or P3HB/PEG (50:50) solutions in dichloromethane (400 mg, $40 \mathrm{ml}$ ) using Büchi B-290 Spray dryer (BÜCHI Laboratory Equipment, Switzerland, Flawil). In brief, a polymer solution was sprayed through a nozzle (diameter of $0.7 \mathrm{~mm}$ ) at a feed rate $1.5 \mathrm{ml} / \mathrm{min}$ at the inlet temperature $75^{\circ} \mathrm{C}$. The value of the aspirator (current of argon) was supported at the maximum gas flow rate $35 \mathrm{~m}^{3} / \mathrm{h}$.

CEF-loaded microparticles were prepared by spray drying water/oil-emulsions. To the solutions of polymer were added an aqueous solution of CEF $(80 \mathrm{mg} / \mathrm{ml})$. And those emulsions were homogenized using sonication at a power of $6 \mathrm{~W}$ for $2 \mathrm{~min}$. The obtained homogeneous emulsions were continuously stirred (700 rpm) and sprayed at the described above parameters.

\section{Characterization of microparticles}

\section{Morphological analysis}

Scanning electron microscopy (SEM) analysis of the prepared CEF-loaded microparticles was carried out using the S-5500 (Hitachi, Japan). The samples were sputter-coated with 
platinum using an electrical potential of $2.0 \mathrm{kV}$ at $25 \mathrm{~mA}$ for 6 min with a sputter coater $\mathrm{K} 550 \mathrm{X}$ (Emitech, Quorum Technologies Ltd., UK).

\section{Process yield (\%)}

The processing yields were defined as the percentage of the weight of microparticles $\left(\mathrm{W}_{\mathrm{m}}\right)$ compared to the weight of polymer $\left(\mathrm{W}_{\mathrm{p}}\right)$ in the initial solution as shown in Eq. (1):

$$
\text { Yield }(\%)=\frac{w_{m}}{W_{p}} \times 100
$$

\section{Measurement of the particle size and zeta potential}

About $5 \mathrm{mg}$ of each sample was suspended in bidistilled water, sonicated at $6 \mathrm{~W}$ for 1 min. The size distribution of the microparticles was determined with the principle of laser diffraction using a particle size analyzer at $25^{\circ} \mathrm{C} \pm 1{ }^{\circ} \mathrm{C}$ (Zetasizer Nano ZS, Malvern Ltd., UK). The average particle size measurements were studied in triplicates. The surface charge of microparticles was characterized by value of zeta potential, which was determined by the electrophoretic mobility of the particles in the suspension on Zetasizer Nano ZS.

\section{Drug encapsulation efficiency}

The amount of drug inclusion in the polymeric microparticles was determined by spectrophotometric analysis. The CEF-loaded $\mathrm{MP}_{\mathrm{SD}}$ were dissolved in dichloromethane and water was then added in a ratio 1:1. The resulting emulsion was mechanically stirred on a shaker to extract the CEF into water. An aqueous phase containing ceftriaxone was then taken and optical density measurements were made. $\mathrm{CEF}$ encapsulation efficiency of $\mathrm{MP}_{\mathrm{EM}}$ was measured according to the procedure described previously (Murueva et al., 2013).

The quantity of CEF loaded into microparticles was determined on a UV-Vis spectrophotometer Cary 60 (Agilent Technologies, Selangor, Malaysia) by measuring the UV-Vis absorbance at $240 \mathrm{~nm}$ using pre-built calibration graphs. The experiment was carried out in triplicates.

The encapsulation efficiency (EE) was defined as the percentage of the weight of drug in microparticles $\left(\mathrm{W}_{\mathrm{m}}\right)$ compared to the initial weight of $\operatorname{drug}\left(\mathrm{W}_{\mathrm{i}}\right)$ as shown in Eq. (2):

$$
E E(\%)=\frac{W_{m}}{W_{i}} \times 100
$$

\section{In vitro ceftriaxone release studies}

The controlled drug release from CEF-loaded microparticles was carried out in vitro. The microparticles were initially sterilized by UV radiation for 40 minutes and placed in a sterile centrifuge tubes, containing $10 \mathrm{ml}$ of phosphate-buffered saline (PBS, pH 7.4). Those centrifuge tubes were exposed to thermostat at $37{ }^{\circ} \mathrm{C}(\mathrm{n}=3)$. Microparticles were settled by centrifugation (10000 rpm, $10 \mathrm{~min}$ ) and samples subjected to determine the amount of the drug released at different intervals. $1 \mathrm{ml}$ samples were withdrawn from the solution to observe the change in CEF 
concentration by UV-Vis spectroscopy (Agilent Technologies, Malaysia). The amount of volume used from centrifuge tubes was replaced with fresh phosphate buffer. The amount of CEF in the supernatant was determined at $240 \mathrm{~nm}$. Triplicate measurements were performed during every analysis.

\section{Cell culture}

\section{In vitro cytotoxicity assay}

The determination of the possible toxicity of PHA microparticles of different chemical composition was investigated in experiments using the NIH 3T3 mouse fibroblast line, which was plated on microparticles $(5 \times 103$ cells $/ \mathrm{cm} 2)$ placed in 24 -well plates as described in Nakoaka R. [Nakaoka R. et al., 2002]. The suspensions of microparticles in phosphate buffer were used at a concentration of $2 \mathrm{mg} / \mathrm{ml} ; 100 \mu \mathrm{l}$ of a suspension of particles of each type were introduced into 24-well culture plates (Corning, USA). Polystyrene plates (Corning, USA) were used as a control.

Culturing the fibroblasts was performed according to a standard procedure in a DMEM medium [Фрешни Р.Я., 2010], containing a 10\% solution of embryonic bovine serum, a solution of antibiotics (streptomycin $100 \mu \mathrm{g} / \mathrm{ml}$, penicillin $100 \mathrm{U} / \mathrm{ml}$ ) in a $\mathrm{CO} 2$ incubator (New Brunswick Scientific, USA) at 5\% CO2 in the atmosphere and $37^{\circ} \mathrm{C}$. The medium was replaced every three days.

The viability of NIH 3T3 cells was evaluated in a reaction with MTT. For this purpose, $50 \mu \mathrm{l}$ of a $5 \%$ MTT solution and $950 \mu \mathrm{l}$ of the total nutrient medium were added to the well with each type of polymer. After 3.5 hours of culture the medium with the MTT solution was replaced with DMSO to dissolve the formed MTT formazan crystals. After 30 minutes the supernatant was transferred to a 96-well plate (Corning, USA) and the optical density was measured at a wavelength of $540 \mathrm{~nm}$ on the Bio-Rad 680 microplate photometer (Bio-Rad Laboratories Inc., США) . The number of cells was estimated from the calibration schedule.

\section{Microbiological evaluation}

The antibacterial activity of antibiotic-loaded microparticles (Ceftriaxone) was determined using the disc-diffusion method for Gram-positive bacteria Staphylococcus aureus and Gram-negative bacteria Escherichia coli. This method is based on the diffusion of an antibacterial drug from the carrier into a dense nutrient medium and inhibition of the growth zone of the culture [МУК 4.2.1890-04]. Susceptibility disk of ceftriaxone was used as the control (with a drug content of $0.03 \mathrm{mg}$, BioRad, France). The concentration of encapsulated ceftriaxone when microparticles were introduced into the cell culture as a suspension was 0.3 mg. 
The Mueller-Hinton medium (BioRad, France) was diluted in distilled water ( $25 \mathrm{ml}$ per dish) and heated until completely dissolved. The nutrient medium was then sterilized by autoclaving at $1 \mathrm{~A}$ and $121^{\circ} \mathrm{C}$ for 15 minutes. Petri dishes were filled with medium on a horizontal surface so that the thickness of the agar layer in the dish was on average $4 \mathrm{~mm}$, and left at room temperature until completely solidified.

To determine the sensitivity of the microorganisms, an inoculum corresponding to a density of 0.5 according to the McFarland standard and containing about $1.5 \times 108 \mathrm{CFU} / \mathrm{ml}$ was used. The inoculation was performed with sterile cotton swabs with uniform strokes. After 15 minutes, in the middle of the Petri dish, vertical wells with a diameter of $15 \mathrm{~mm}$ were made in agar and the microparticle suspension in physiological saline in a volume of $100 \mu$ was excavated in them. The application of the disks was performed on agar without the formation of wells using sterile tweezers. Upon completion Petri dishes were left in a thermostat at $37^{\circ} \mathrm{C}$. After a day, the diameter of the culture growth retardation zones was measured.

\section{Results and discussion}

In this study, polymer-based microparticles are used to improve the therapeutic properties of CEF and render them safer. PHB- and PHB-PEG-based microparticles were prepared by two methods: a double emulsification technique and spray-drying (abbreviations are presented in Table 2).

\section{Characterization of microparticles}

Microparticles size and polydispersity index were determined using dynamic light scattering by analyzer of Zetasizer Nano ZS. Results revealed that microparticles had a size range from 0.74 to $1.55 \mu \mathrm{m}$ (emulsion method) and from 3.84 to $6.51 \mu \mathrm{m}$ (spray-dried); a negative zeta potential ranging from -17.8 to $-28.0 \mathrm{mV}$ and from -37.5 to $-95.7 \mathrm{mV}$ respectively (Table 2 ). It is advisable to assume that the difference in $\zeta$ potentials is probably due to the adsorption on the surface of the microparticles of polyvinyl alcohol, which is used in the emulsion method and absent in the spray drying method.

Table 2 - Sample composition and characterization of the obtained microparticles

\begin{tabular}{|c|c|c|c|c|c|c|}
\hline Samples & $\begin{array}{c}\text { Process } \\
\text { yield, } \%\end{array}$ & $\begin{array}{c}\text { Particle } \\
\text { size, } \mu \mathrm{m}\end{array}$ & PdI & $\begin{array}{c}\text { Zeta } \\
\text { potential, } \\
\mathrm{mV}\end{array}$ & $\begin{array}{c}\text { Encapsulation } \\
\text { efficiency, } \%\end{array}$ \\
\hline \multicolumn{7}{|c|}{ Emulsification technique } \\
\hline $\mathrm{P}_{\mathrm{EM}}$ & P3HB & 72.5 & $0.74 \pm 18.82$ & $0.187 \pm 0.063$ & $-17.8 \pm 0.3$ & - \\
\hline $\mathrm{PP}_{\mathrm{EM}}$ & P3HB-PEG & 39.0 & $1.55 \pm 0.05$ & $0.167 \pm 0.037$ & $-28.0 \pm 0.4$ & - \\
\hline $\mathrm{PC}_{\mathrm{EM}}$ & P3HB- CEF & 71.4 & $0.89 \pm 0.02$ & $0.250 \pm 0.003$ & $-22.3 \pm 0.6$ & 66.0 \\
\hline
\end{tabular}




\begin{tabular}{|c|c|c|c|c|c|c|}
\hline PPC $_{\mathrm{EM}}$ & $\begin{array}{c}\text { P3HB-PEG- } \\
\text { CEF }\end{array}$ & 26.8 & $1.55 \pm 0.01$ & $0.297 \pm 0.027$ & $-25.0 \pm 0.1$ & 60.5 \\
\hline \multicolumn{7}{|c|}{ Spray-drying } \\
\hline $\mathrm{P}_{\mathrm{SD}}$ & P3HB & 33.2 & $6.51 \pm 0.47$ & $0.211 \pm 0.058$ & $-95.7 \pm 0.6$ & - \\
\hline $\mathrm{PP}_{\mathrm{SD}}$ & P3HB-PEG & 51.1 & $4.06 \pm 0.38$ & $0.318 \pm 0.192$ & $-37.5 \pm 2.2$ & - \\
\hline $\mathrm{PC}_{\mathrm{SD}}$ & P3HB- CEF & 85.7 & $4.21 \pm 0.70$ & $0.260 \pm 0.118$ & $-48.0 \pm 1.4$ & 50.6 \\
\hline $\mathrm{PPC}_{\mathrm{SD}}$ & $\begin{array}{c}\text { P3HB-PEG- } \\
\text { CEF }\end{array}$ & 34.5 & $3.84 \pm 0.27$ & $0.224 \pm 0.058$ & $-38.7 \pm 1.2$ & 47.0 \\
\hline
\end{tabular}

It was found that the addition of PEG leads to a decrease in process yield, an increase in average diameter and zeta-potential of $\mathrm{MP}_{\mathrm{EM}}$. On the other hand, the availability of PEG in $\mathrm{MP}_{\mathrm{SD}}$ resulted in a decrease of size and an increase of zeta-potential. Thus, CEF loading did not significantly affect size characteristics and process yield of MPs. The deposition efficiency was about $60 \%$ and $50 \%$ for $\mathrm{MP}_{\mathrm{EM}}$ and $\mathrm{MP}_{\mathrm{SD}}$, respectively.

Fig.1 shows the SEM images of CEF-loaded MPs. It was found that additional of PEG had important influence on the surface morphologies and microstructures of MPs. So PC $\mathrm{SD}$ had a smooth surface, while the surface of the $\mathrm{PPC}_{\mathrm{SD}}$ was rough with availability of small pores.
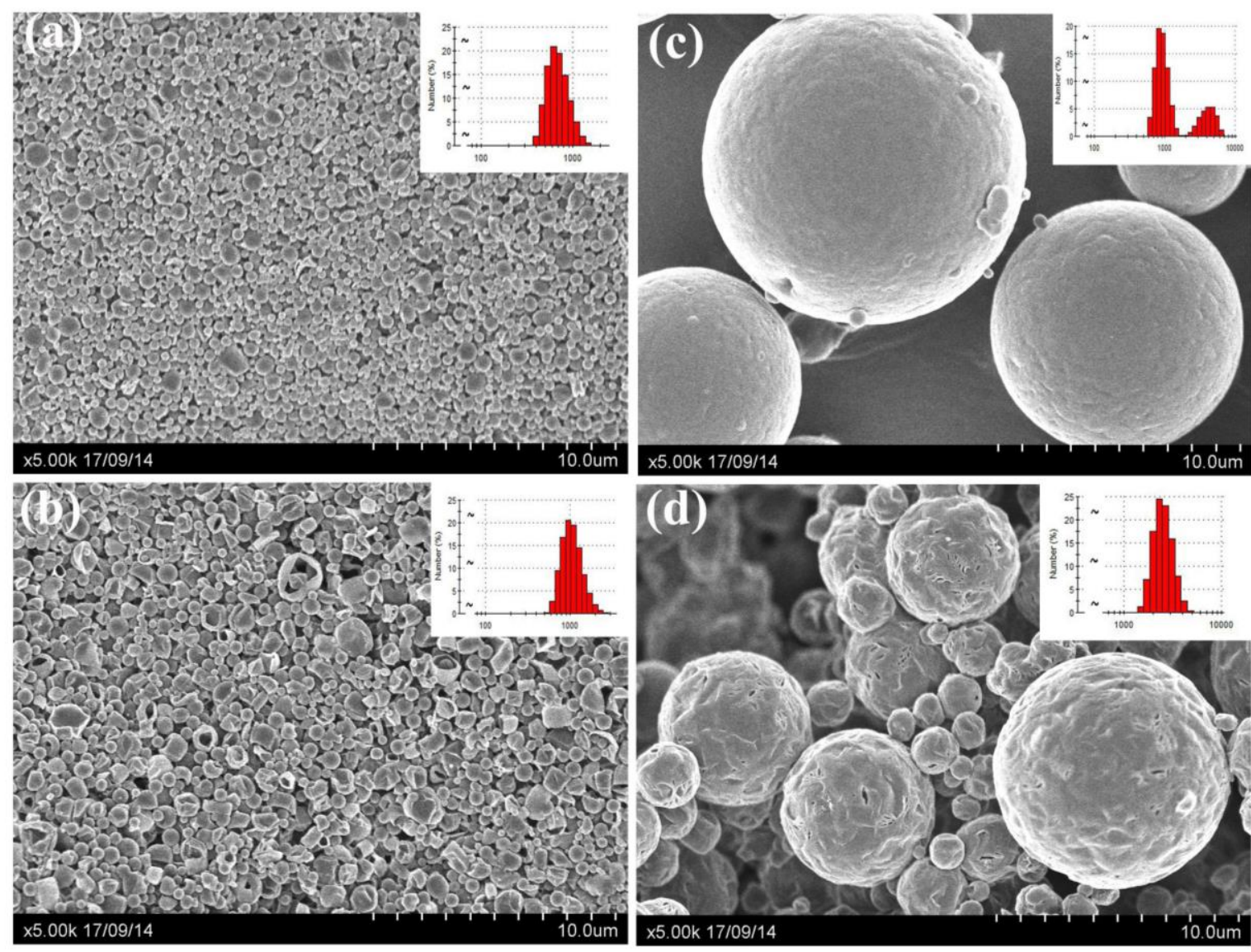

Figure 1. SEM images and size distribution of microparticles with CEF: $\mathrm{a}-\mathrm{PC}_{\mathrm{EM}} ; \mathrm{b}-\mathrm{PPC}_{\mathrm{EM}} ; \mathrm{c}-\mathrm{PC}_{\mathrm{SD}} ; \mathrm{d}-\mathrm{PPC}_{\mathrm{SD}}$ (all images share the same scale bar) 
Consequently, the addition of PEG as surfactant most likely led to a decrease in the amorphization of substances in the spray-drying process. A similar effect of the PEG use in spray drying was noted in the review of Paudel et al. [2013]. Moreover, PPC $_{\mathrm{EM}}$ were also characterized by a change in the structure upon the addition of PEG. However, in this case PEG was dissolved in the process of microparticles preparation, leading to the formation of deformed particles. According to Li et al. [2009] PEG influenced the formation of pores on the surface of microparticles, but only with the correct ratio PHB-PEG. When the ratio of PEG was excessively high, the erosion and dissolving effect were so strong that it was impossible to integrate microparticles [Li et al., 2009]. On the whole, from the micrographs of Fig. 1 it could be seen that the $\mathrm{MP}_{\mathrm{SD}}$ were spherical in shape and significantly larger in size than the $\mathrm{MP}_{\mathrm{EM}}$.

\section{Study of the ceftriaxone release from MPs in vitro}

$\mathrm{CEF}$ release profiles from $\mathrm{MP}_{\mathrm{EM}}$ and $\mathrm{MP}_{\mathrm{SD}}$ are presented in Fig. 2. The mean amount of CEF released from $\mathrm{PC}_{\mathrm{SD}}$ and $\mathrm{PPC}_{\mathrm{SD}}$ was $51.3 \%$ and $39.1 \%$ respectively in the first $6 \mathrm{~h}$ and all the amount of drug from these formulations was released at approximately $150 \mathrm{~h}$ (Fig. 2a).

Otherwise, $\mathrm{CEF}$ release from $\mathrm{PC}_{\mathrm{EM}}$ and $\mathrm{PPC}_{\mathrm{EM}}$ microparticles was seen retardant- the effect compared with $\mathrm{PC}_{\mathrm{SD}}$ and $\mathrm{PPC}_{\mathrm{SD}}$ (Fig. 2b). In the first $6 \mathrm{~h} 9.4 \%$ and $2.6 \%$ of the CEF was released from the $\mathrm{PPC}_{\mathrm{EM}}$ and $\mathrm{PC}_{\mathrm{EM}}$, respectively. Around $15 \%$ of drug was released within $48 \mathrm{~h}$ for $\mathrm{PPC}_{\mathrm{EM}}$, while $4 \%$ was released in the same period from $\mathrm{PC}_{\mathrm{EM}}$. It is obvious that the drug release rate was increased when PEG was introduced into P3HB solution at emulsification technique. Likely, incorporation of PEG increased the release rate due to its high hydrophilicity and water solubility (Li et al., 2009, Parra et al., 2006; Rodrigues et al., 2005). As soluble filler, dissolution of PEG upon contact with a release medium would provide more channels for drug diffusion, inducing more drug molecules to be released. After 312 h, $34 \%$ and $10 \%$ of CEF were released from the $\mathrm{PPC}_{\mathrm{EM}}$ and $\mathrm{PC}_{\mathrm{EM}}$ microparticles, respectively.

Earlier scientific literature reports that in the absence of biologic factors (enzymes, cells) the P3HB does not hydrolyze into carbon chains (Köse et al., 2003). This is due to the very high crystallinity and hydrophobic nature of polymer. Therefore, the rate of drug diffusion was substantially higher than that of polymer degradation, so the release profiles are more dependent on drug diffusion rather than on polymer degradation. 

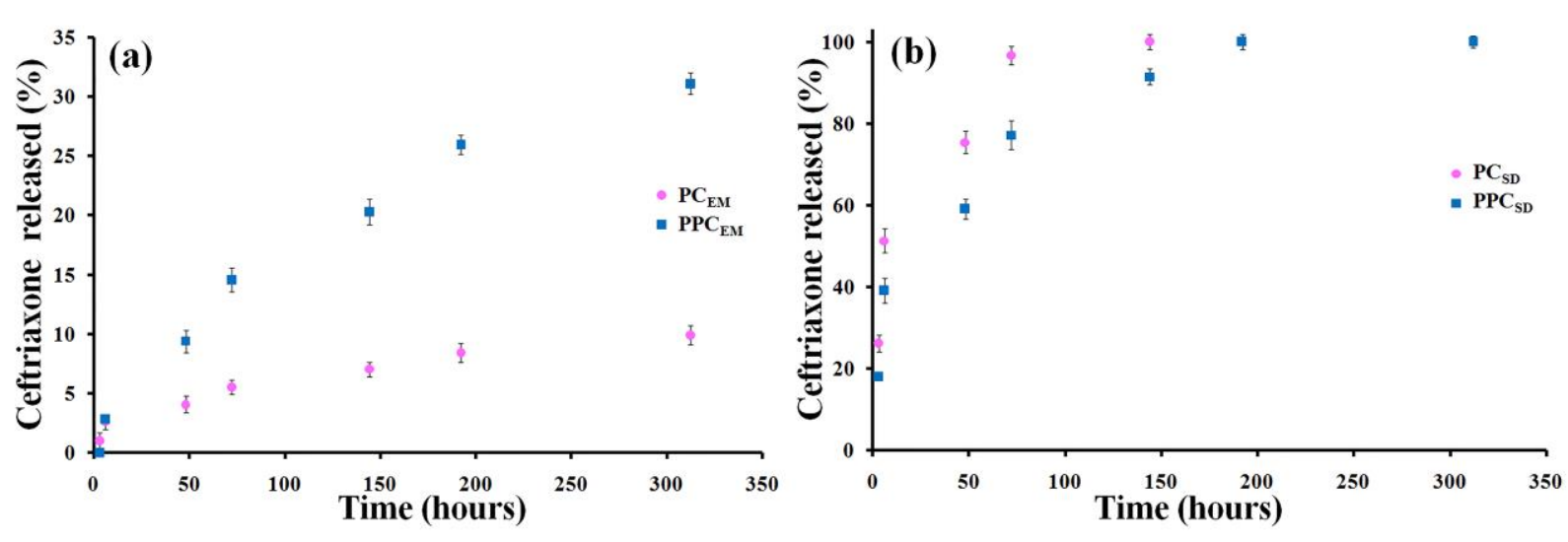

Figure 2. CEF release profile from (•) PC and ( $\bullet$ PPC microparticles prepared by emulsification technique (a) and spray-drying (b)

The difference in the total release of CEF from $\mathrm{MP}_{\mathrm{EM}}$ and $\mathrm{MP}_{\mathrm{SD}}$ is most likely due to the fact that the drug molecules had different variants of localization in the particles and, as a consequence, different release rates.

$\mathrm{MP}_{\mathrm{EM}}$ had a relatively low total drug release, which could be explained by the hydrophobicity of $\mathrm{P} 3 \mathrm{HB}$ and dense packing of polymer chains in the transformation process of microdroplets into microparticles as a result, most of the drug molecules being inside the particles.

At the same time, it was found that the increase of $\mathrm{CEF}$ release rates from $\mathrm{PC}_{\mathrm{SD}}$ and $\mathrm{PPC}_{\mathrm{SD}}$ can be related to the fact that the part of CEF solidifies separately during co-spray drying polymers. The remaining part of CEF may most probably be located near the surface. Therefore, drug molecules close to the surface leave the matrix very easily. Most likely, the drug release from $\mathrm{PC}_{\mathrm{SD}}$ and $\mathrm{PPC}_{\mathrm{SD}}$ in the first 3-6 hours is associated with solubilization of free CEF. The next phase of CEF release (48-312 h) was dominated by the diffusion mechanisms associated with the drug release from the surface structures of microparticles.

In this way, these results confirm that it is possible to control the drug release by varying the method of preparation and chemical composition of MPs.

\section{In vitro cytotoxicity assay}

\section{Microbiological evaluation}

The antibacterial activity of CEF, microparticles and empty particles was investigated against various bacterial strains including Escherichia coli and Staphylococcus aureus using the disk-diffusion method with the zones of inhibition (ZOI) to be measured (Fig. 4).

Fig. 4 - Antibacterial activity of microparticles against E.coli and St.aureus 
The antibacterial activity was compared against susceptibility disk of CEF. The antibacterial efficiency of free $\mathrm{CEF}$ was higher against $E$. coli in comparison to $S$. aureus, which is consistent with these minimum inhibitory concentrations. Herewith samples obtained by spray-drying showed higher antibacterial activity in comparison to disk (Table 3).

The only sample obtained by the emulsification technique, $\mathrm{PPC}_{\mathrm{EM}}$ insignificantly suppressed growth of the more sensitive E. coli, while the remaining samples did not inhibit both E. coli and S. aureus (Table 3, Fig. 4). Probably this is due to the fact that the concentration of released $\mathrm{CEF}$ from $\mathrm{PC}_{\mathrm{EM}}$ and $\mathrm{PPC}_{\mathrm{EM}}$ was insufficient to suppress the growth of colonies. It is also worth noting that there have been some case studies of antibiotic-loaded PH3B, while their applications for cephalosporins cannot be easily found. For instance, Vilos et al. (2012) developed ceftiofur-loaded PHBV microparticles, which showed a slight bacterial inhibition during the first $5 \mathrm{hrs}$ and a delay in the onset of the bacterial exponential growth E. coli.

Table 3 - Antibacterial activity of in vitro microparticles against E.coli and St.aureus. Diameter of inhibition zones $(\mathrm{mm})$

\begin{tabular}{|c|c|c|c|c|c|c|c|c|c|}
\hline $\begin{array}{c}\text { Test } \\
\text { strain }\end{array}$ & $\begin{array}{c}\text { Disk of } \\
\mathrm{CEF}\end{array}$ & $\mathrm{P}_{\mathrm{EM}}$ & $\mathrm{PP}_{\mathrm{EM}}$ & $\mathrm{PC}_{\mathrm{EM}}$ & $\mathrm{PPC}_{\mathrm{EM}}$ & $\mathrm{P}_{\mathrm{SD}}$ & $\mathrm{PP}_{\mathrm{SD}}$ & $\mathrm{PC}_{\mathrm{SD}}$ & $\mathrm{PPC}_{\mathrm{SD}}$ \\
\hline E. coli & $20.0 \pm 0.3$ & - & - & - & $5.1 \pm 0.2$ & - & - & $26.5 \pm 0.2$ & $23.3 \pm 0.1$ \\
\hline S. aureus & $6.2 \pm 0.8$ & - & - & - & - & $16.0 \pm 0.3$ & $12.0 \pm 0.1$ & $26.7 \pm 0.2$ & $22.0 \pm 0.3$ \\
\hline
\end{tabular}

As a control, the activity of unloaded $\mathrm{P} 3 \mathrm{HB}$ microparticles and the physiological solution, used for suspending the microparticles was checked. In both cases, no growth inhibition was observed in the analyzed cultures, which excludes the possibility of the influence of the chemical composition of the polymer and the medium used for suspending on the results of the efficacy of the encapsulated form of ceftriaxone (Table 3). The results obtained correlate with the data presented in the paper by Hema et al., in which the researchers confirmed the absence of antibacterial activity of films based on P (3HB), P (3HB-co-3HV) (6 mol\%) and P (3HB-co-4HB ) (70 mol\%) with respect to E. coli and S. aureus [Hema R. et al., 2013].

\section{Conclusion}

The outcome of this study is the development and characterization of CEF-loaded polymeric microparicles. P3HB- and P3HB-PEG-based microparticles were prepared by two methods: a double emulsification technique and spray-drying. The deposition efficiency of CEF 
was about $60 \%$ and $50 \%$ for $\mathrm{MP}_{\mathrm{EM}}$ and $\mathrm{MP}_{\mathrm{SD}}$, respectively. It is shown that the surfactants used in the emulsion method affect on the electrophoretic activity of the microparticles. Therefore, the zeta potential of $\mathrm{MP}_{\mathrm{EM}}$ was lower than that of $\mathrm{MP}_{\mathrm{SD}}$ by an average of 3 times. The addition of PEG to the P3HB solutions had important influence on the surface morphologies and microstructures of microparticles; however, the effect on drug release rate was clearly expressed only for $\mathrm{MP}_{\mathrm{EM}}$. The total release of $\mathrm{CEF}$ from $\mathrm{MP}_{\mathrm{EM}}$ and $\mathrm{MP}_{\mathrm{SD}}$ had significant difference, which is most likely related to the localization of the drug molecules in the microparticles. So, the CEF release of $\mathrm{MP}_{\mathrm{SD}}$ reached $100 \%$ after 150 hours, while for $\mathrm{MP}_{\mathrm{EM}}$ the total release of CEF did not exceed $34 \%$ for the entire observation period.

The bactericidal potential of $\mathrm{MP}_{\mathrm{EM}}$ and $\mathrm{MP}_{\mathrm{SD}}$ with and without $\mathrm{CEF}$ was investigated against Escherichia coli and Staphylococcus aureus by disk-diffusion method. MP $\mathrm{SD}_{\text {with CEF }}$ showed higher antibacterial activity (ZOI over $22.0 \mathrm{~mm}$ ) in comparison to $\mathrm{MP}_{\mathrm{EM}}$ (ZOI not more than $5.1 \mathrm{~mm}$ ). Thus, using various methods, the possibility of inclusion CEF with satisfactory indicators of the effectiveness of encapsulation, drug outflow and preservation of therapeutic activity in vitro into the composition of P3HB-carriers is shown, which allows to conclude that this class of polymers is promising for the development of long-acting dosage forms. 